\section{Avaliação da literacia para a saúde de pacientes portadores de diabetes acompanhados em um ambulatório público}

\author{
Assessment of health literacy in diabetic patients \\ followed at a public outpatient clinic
}

\author{
Evaluación de la alfabetización en salud con \\ pacientes diabéticos, cuyo seguimiento se realiza \\ en un ambulatorio público
}

Ana Luiza Braz Pavão 1

Guilherme Loureiro Werneck 2

Luis Saboga-Nunes 3

Rosane Aparecida de Sousa 4 doi: $10.1590 / 0102-311 \times 00084819$

\section{Resumo}

Literacia para a saúde (LS), ou literacia em saúde, pressupõe o conhecimento, a motivação e as competências dos indivíduos para acessarem, compreenderem, avaliarem e aplicarem as informações sobre saúde, a fim de fazer julgamentos e tomar decisões na vida diária, relacionadas aos cuidados de saúde, à prevenção de doenças e à promoção de saúde, para manter ou melhorar a sua qualidade de vida. O objetivo foi medir o nível de LS e seus fatores associados: sexo, idade, escolaridade, renda, cor da pele, autoavaliação do estado de saúde, tipo de diabetes e presença de comorbidades. Foram avaliados 107 adultos portadores de diabetes acompanhados em um ambulatório público, na cidade do Rio de Janeiro, Brasil. Não foram incluídos pacientes de primeira vez, pacientes sem diagnóstico de diabetes ou com limitações de visão ou audição. A LS foi avaliada pela versão brasileira do questionário European Health Literacy Survey (HLS-EU-BR). Modelos de regressão logística ordinal simples e múltiplos foram construídos, considerando-se, como variável dependente, os quatro níveis de LS. As associações foram expressas na forma de odds ratio (OR). Cerca de 95\% da amostra apresentou nível de LS ruim ou limitado (94,8\%; IC95\%: 90,3-99,3). Sexo feminino, idades mais avançadas e menor escolaridade estiveram associados a uma menor chance de ter um nivel de LS excelente. No modelo ajustado, apenas a variável escolaridade permaneceu estatisticamente significativa em relação ao seu efeito sobre a LS (OR ajustado= 0,41; IC95\%: 0,17-0,98; $p<0,05)$. Escolaridade foi a característica que esteve mais fortemente relacionada ao nível de LS.

Inquéritos e Questionários; Diabetes Mellitus; Letramento em Saúde

\author{
Correspondência \\ A. L. B. Pavão \\ Instituto de Comunicação e Informação Científica e Tecnológica \\ em Saúde, Fundação Oswaldo Cruz. \\ Av. Brasil 4365, Rio de Janeiro, RJ 21040-900, Brasil. \\ ana.pavao@icict.fiocruz.br

\footnotetext{
1 Instituto de Comunicação e Informação Científica e Tecnológica em Saúde, Fundação Oswaldo Cruz, Rio de Janeiro, Brasil.

2 Instituto de Medicina Social, Universidade do Estado do Rio de Janeiro, Rio de Janeiro, Brasil.

3 Institute of Sociology, University of Education, Freiburg, Germany.

4 Departamento de Serviço Social, Universidade Federal do Triângulo Mineiro, Uberaba, Brasil.
} 


\section{Introdução}

A literacia para a saúde (LS), ou literacia em saúde, é a expressão que vem sendo usada em estudos, na língua portuguesa, que se referem à literacia no campo da saúde ou à literacia aplicada à área da saúde 1,2,3. Em inglês, é traduzida pelo termo "health literacy". Há também, alguns estudos recentes que utilizam como sinônimos para LS: letramento em saúde, alfabetização em saúde ou alfabetismo em saúde 4,5,6,7.

Existem diversas definições para LS na literatura. As mais comumente citadas são a da Associação Médica Americana (AMA) 8, a do Instituto de Medicina dos Estados Unidos (IOM) 9 e a da Organização Mundial da Saúde (OMS) 10. Segundo a definição da AMA 8 (p. 552), LS é "o conjunto de habilidades, incluindo a habilidade de executar leituras básicas e tarefas numéricas necessárias para funcionar no ambiente de cuidado de saúde". A definição proposta pelo IOM ${ }^{9}$ (p. 32) descreve LS como "o grau com que indivíduos possuem a capacidade de obter, processar e compreender informações básicas sobre saúde, bem como os serviços necessários para se tomar decisões apropriadas em saúde". Segundo a definição proposta pela OMS 10 (p. 10), a LS representa "as habilidades cognitivas e sociais que determinam a motivação e a capacidade de os indivíduos obterem acesso a compreenderem e usarem informações de forma a promover $e$ manter uma boa saúde".

As diferentes definições foram analisadas posteriormente por alguns autores. Pleasant \& Kuruvilla 11 descreveram duas abordagens principais, adotadas pelas diferentes definições de LS, que são a abordagem clínica e a abordagem de saúde pública. A abordagem clínica foi desenvolvida principalmente nos Estados Unidos com a finalidade de ajudar os médicos a melhorarem a comunicação de suas prescrições e para ajudar os pacientes a melhor compreenderem e cumprirem os regimes terapêuticos. Já a abordagem de saúde pública, relaciona a LS com a promoção de saúde, o marketing social das intervenções de saúde pública, a educação e o empoderamento, considerando a LS igualmente importante tanto na esfera pública como nos ambientes de cuidado de saúde. Os autores consideram que ambas tendem a se concentrar em algum aspecto das capacidades individuais de obter, compreender, avaliar e usar a informação a fim de promover a tomada de decisão relacionada à saúde, de melhorar a saúde e/ou de reduzir as desigualdades em saúde e sugerem uma abordagem para a LS que inclua tanto a abordagem clínica como a de saúde pública.

Nutbeam 12 identificou duas abordagens existentes para a LS: a primeira vista como um fator de risco e a segunda vista como um recurso, um bem, um ativo (asset). A primeira está relacionada aos estudos conduzidos nos Estados Unidos, que consideram LS um conjunto de capacidades individuais de literacia, atuando como um fator mediador entre a saúde e a tomada de decisão clínica. A segunda está relacionada à definição adotada pela OMS, segundo a qual a LS não é apenas um conjunto de capacidades funcionais, mas consiste no conjunto de habilidades que permite aos indivíduos participarem mais plenamente da sociedade e exercerem um maior grau de controle sobre os eventos da vida diária. Possui origem no âmbito da saúde pública e da promoção de saúde.

Mais recentemente, em 2012, Sorensen et al. 13, numa tentativa de consolidar o conhecimento existente sobre o tema, realizaram uma revisão sistemática dos modelos teóricos e definições de LS e propuseram um modelo teórico-conceitual para a LS que englobasse tanto a perspectiva da saúde pública como a perspectiva individual. Segundo os autores, a LS deve ser entendida como um recurso, um bem que visa promover o empoderamento dos indivíduos nos cenários do cuidado de saúde, prevenção de doenças e promoção de saúde. De acordo com o modelo proposto, a "LS está intimamente ligada à literacia e pressupõe o conhecimento, a motivação e as competências dos indivíduos para acessarem, compreenderem, avaliarem e aplicarem as informações sobre saúde, a fim de fazer julgamentos e tomar decisões na vida diária, relacionadas aos cuidados de saúde, à prevenção de doenças e à promoção de saúde, para manter ou melhorar a sua qualidade de vida durante o curso da vida" 13 (p. 3).

Existem diversos instrumentos desenvolvidos para medir o construto da LS, como: Rapid Estimate of Adult Literacy in Medicine (REALM), Test of Functional Health Literacy in Adults (TOFHLA) e Newest Vital Sign (NVS). A maioria deles foram criados na década de 1990 e início dos anos 2000, com a finalidade de avaliarem a literacia funcional, que é a dimensão da LS que se relaciona às habilidades individuais relacionadas à leitura, à escrita e à compreensão da linguagem escrita $9,14,15$. Foram desenvolvidos no contexto de uma abordagem clínica da LS, a fim de permitirem medidas mais rápidas desse construto, adequando o seu uso aos serviços de saúde 11,16. Nos últimos anos, outros 
instrumentos vêm sendo propostos na tentativa de incorporar a complexidade do construto da LS e de permitir a sua avaliação, não só em nível individual, mas também sob a perspectiva populacional. Dentre eles, destacam-se o European Health Literacy Survey (HLS-EU-Q), desenvolvido a partir da revisão sistemática conduzida por Sorensen et al. 13, e o Health Literacy Questionnaire, ambos utilizando a LS autorreferida pelos indivíduos como método de avaliação 16.

Diversos estudos têm demonstrado associação entre baixo nível de LS e piores indicadores de saúde, tais como: mortalidade precoce, maior incidência de doenças crônicas, menor uso de serviços preventivos, maior uso de serviços de emergência e aumento nas internações hospitalares, consumo de medicamentos mais elevado e frequente e autoavaliação negativa do estado de saúde, entre outros 9,12,17,18,19. Além de afetar diretamente a saúde, a LS está fortemente associada a indicadores socioeconômicos, como a escolaridade, a raça/etnia, a idade, a renda e o nível cultural do indivíduo 17,20,21. Um estudo conduzido em indivíduos diabéticos demonstrou que níveis inadequados de LS estiveram associados a um pior controle glicêmico da doença e a maiores taxas de retinopatia diabética, independentemente do efeito de variáveis sociodemográficas 22.

Seguindo o modelo conceitual proposto por Sorensen et al. 13, o objetivo do presente estudo será medir o nível de LS e seus fatores associados (sexo, idade, escolaridade, renda, cor da pele, autoavaliação do estado de saúde, tipo de diabetes e presença de comorbidades), por meio da aplicação da versão brasileira do questionário European Health Literacy Survey (HLS-EU-BR) 23, numa população de pacientes, portadores de diabetes, atendidos em um ambulatório público. Optou-se por aplicar o questionário em uma população de diabéticos porque se trata de uma doença crônica de alta prevalência e elevado impacto na morbimortalidade e que, acredita-se, pela complexidade do seu tratamento e do controle da doença, que a LS deva exercer um impacto importante no manejo da doença e na prevenção de suas complicações. Os autores esperam encontrar um baixo nível de LS entre os indivíduos entrevistados.

\section{Metodologia}

Estudo transversal, tipo inquérito, que consistiu na aplicação do questionário HLS-EU-BR, uma adaptação validada para o Brasil do questionário HLS-EU-PT, traduzido para o português de Portugal em 2014, e que deriva do instrumento original HLS-EU-Q, em inglês 13,23,24.

O instrumento original HLS-EU-Q foi elaborado a partir de modelo teórico-conceitual desenvolvido pelo Consórcio Europeu de Literacia em Saúde, descrito em revisão sistemática publicada por Sorensen et al. 2012 13, que identifica 12 subdimensões da LS, relacionadas às competências dos indivíduos para acessar, compreender, avaliar e aplicar as informações sobre saúde, relacionadas aos cuidados de saúde, à prevenção de doenças e à promoção de saúde 20. É composto por 47 perguntas com respostas na forma de escala tipo Likert, que totalizam 50 pontos. De acordo com a pontuação obtida, o nível de literacia é determinado, da seguinte maneira: inadequado ( 0 a 25 pontos), problemático (entre 26 e 33 pontos), suficiente (entre 34 e 42 pontos) e excelente (entre 43 e 50 pontos).

Esse instrumento foi construído a partir de questões, relacionadas às dimensões 13 de prevenção de doença, promoção de saúde e cuidado de saúde, que medissem as dificuldades percebidas pelos indivíduos em realizar tarefas relevantes relacionadas à saúde, tais como: compreender o que o médico diz; avaliar se a informação sobre doença que aparece na mídia é de confiança; encontrar informações sobre como lidar com problemas de saúde mental, como estresse ou depressão; compreender a informação contida nas embalagens dos alimentos e participar de atividades que melhorem a saúde e o bem-estar na comunidade.

Foram incluídos somente pacientes adultos (com 18 anos ou mais), de ambos os sexos, portadores de diabetes tipo 1 ou tipo 2 e que já realizavam acompanhamento para a doença no ambulatório. Não foram incluídos pacientes de primeira vez ou pacientes que não tinham diagnóstico de diabetes. Pacientes com limitações de visão ou audição também não foram incluídos.

O ambulatório de diabetes em que foi conduzido o estudo faz parte do conjunto de clínicas de uma policlínica de assistência pública secundária e que oferece atendimento integral com equipe multiprofissional (médico, enfermeiro, psicólogo, nutricionista, fisioterapeuta, pedagogo e educador físico) para usuários com diagnóstico de diabetes mellitus tipo 1, tipo 2 e diabetes gestacional. 
Durante a realização das entrevistas, que duravam, em média, 30 minutos cada, a pesquisadora se comprometia a explicar os objetivos da pesquisa, como essa seria conduzida e, ao iniciar as perguntas ao entrevistado, mantinha-se na sequência do questionário e buscava sanar as dúvidas que, por ventura, surgiam em relação a alguma pergunta, esclarecendo alguma palavra ou interpretação de difícil compreensão por parte do entrevistado, tendo por objetivo que ele permanecesse motivado até o fim da entrevista e certificando-se, na medida do possível, de que o entrevistado havia compreendido a pergunta. O tempo total de duração da coleta de dados foi de 2 a 3 meses.

Para o cálculo do tamanho amostral, considerou-se a proporção de LS inadequada de 65,9\%, baseada em estudo prévio de avaliação do letramento em saúde de pacientes diabéticos tipo 2 de um ambulatório de endocrinologia de um hospital público brasileiro ${ }^{5}$, para um nível de $95 \%$ de confiança e uma diferença aceitável de $9 \%$. Considerando-se esses parâmetros, o tamanho amostral calculado foi de 107 participantes.

Os pacientes foram recrutados na própria sala de espera do ambulatório e convidados a participar do estudo. De 120 indivíduos recrutados, 2 não aceitaram participar da pesquisa, 4 eram pacientes de outro ambulatório (não portadores de diabetes), 3 eram pacientes de primeira vez, 2 eram menores de 18 anos, e 2 eram acompanhantes de pacientes. Foram entrevistados 107 pacientes no total.

Também foram coletados dados sociodemográficos e de morbidade, tais como: idade, sexo, cor da pele, escolaridade, renda, presença de outras doenças crônicas, tipo de diabetes e autoavaliação do estado de saúde. A renda per capita foi calculada com base no salário mínimo estipulado pelo Governo Federal ( $\mathrm{R} \$ 954,00$, em 1o de janeiro de 2018). As variáveis foram categorizadas da seguinte forma:

- Sexo: masculino e feminino;

- Idade (anos): até 45, 46-64 e 65 ou mais;

- Cor da pele: branca, parda e preta;

- Escolaridade (anos de estudo): menos de 8 e 8 anos ou mais;

- Renda (salários mínimos): até 1 (até R\$ 954,00), > 1 até 2 (acima de R\$954,00 e até R\$1.908,00) e $>2$ (acima de R\$1.908,00);

- Presença de outra morbidade crônica além de diabetes: sim e não.

- Número de morbidades crônicas além de diabetes: nenhuma, até 1 e 2 ou mais;

- Tipo de diabetes: tipo 1 (insulinodependente) e tipo 2 (não insulinodependente);

- Autoavaliação do estado de saúde: muito boa, boa, regular, ruim e muito ruim. Essa variável foi analisada de forma dicotômica, em: boa (unindo as categorias: muito boa e boa) e ruim (unindo as categorias: regular, ruim e muito ruim).

Optou-se por incluir, no estudo, o indicador da autoavaliação do estado de saúde por ser um importante desfecho em saúde e por ser uma medida da percepção de saúde, autorreferida pelos indivíduos e que vem apresentando um efeito direto e independente no indicador da autoavaliação do estado de saúde 20. Como o construto da LS, no presente estudo, será obtido a partir da aplicação de um questionário autorreferido, que também possui um componente de subjetividade assim como o próprio indicador da autoavaliação do estado de saúde, acredita-se que seria importante avaliar também tal indicador nessa população 16 .

Foi realizada análise descritiva dos dados, em que foram calculadas prevalências e seus intervalos de 95\% de confiança (IC95\%). Para a análise da consistência interna do questionário HLS-EU-BR, foi utilizado o teste de alfa de Cronbach. Modelos de regressão logística ordinal simples e múltiplos foram construídos, considerando-se, como variável dependente, os quatro níveis de LS (inadequada, problemática, suficiente e excelente). As associações foram expressas na forma de odds ratio (OR). Apenas as variáveis que apresentaram significância estatística nos modelos simples foram incluídas nos modelos ajustados. Considerou-se valor de p abaixo de 0,05 como estatisticamente significativo. As análises estatísticas foram realizadas no programa Stata/IC, versão 12.0 (https://www.stata.com).

O estudo foi aprovado pelo Comitê de Ética em Pesquisa do Instituto de Medicina Social da Universidade do Estado do Rio de Janeiro (CAAE: 79521717.9.0000.5260), e todos os indivíduos participantes do estudo assinaram um Termo de Consentimento Livre e Esclarecido (TCLE). Foi obtida autorização para o uso do questionário HLS-EU-BR. 


\section{Resultados}

Foi analisada uma amostra de 107 pacientes. Em relação às características sociodemográficas, a maioria era de mulheres (72\%; IC95\%: 63,3-80,6). A idade mínima da amostra era de 18 anos, e a máxima foi de 83 anos, com média de idade de 57,8 anos (desvio padroão - DP: 14,2) e mediana de 61 anos (intervalo interquartil - IQR: 52-68). A maior parte dos indivíduos participantes do estudo possuía renda per capita até 1 salário mínimo (59,4\%; IC95\%: 49,7-69,1) (Tabela 1).

\section{Tabela 1}

Prevalência das características sociodemográficas e de morbidade na população de 107 pacientes diabéticos. Rio de Janeiro, Brasil, 2018.

\begin{tabular}{|c|c|c|}
\hline Características & $\mathbf{n}$ & $\%(I C 95 \%)$ \\
\hline \multicolumn{3}{|l|}{ Sexo } \\
\hline Masculino & 30 & $28,0(19,4-36,7)$ \\
\hline Feminino & 77 & $72,0(63,3-80,6)$ \\
\hline \multicolumn{3}{|l|}{ Faixa etária (anos) } \\
\hline Até 45 & 18 & $16,8(9,6-24,0)$ \\
\hline $46-64$ & 48 & $44,9(35,3-54,4)$ \\
\hline 65 ou mais & 41 & $38,3(29,0-47,7)$ \\
\hline \multicolumn{3}{|l|}{ Cor da pele } \\
\hline Branca & 53 & $49,5(39,9-59,2)$ \\
\hline Parda & 39 & $36,4(27,2-45,7)$ \\
\hline Preta & 15 & $14,0(7,3-20,7)$ \\
\hline \multicolumn{3}{|c|}{ Escolaridade (anos de estudo) } \\
\hline Menos de 8 & 55 & $51,4(41,8-61,0)$ \\
\hline 8 ou mais & 52 & $48,6(39,0-58,2)$ \\
\hline \multicolumn{3}{|c|}{ Renda (salários mínimos) * } \\
\hline Até 1 & 60 & $59,4(49,7-69,1)$ \\
\hline$>1$ até 2 & 23 & $22,8(14,5-31,1)$ \\
\hline$>2$ & 18 & $17,8(10,2-25,4)$ \\
\hline \multicolumn{3}{|l|}{ Tipo de diabetes } \\
\hline Tipo 1 & 25 & $23,4(15,2-31,5)$ \\
\hline Tipo 2 & 82 & $76,6(68,5-84,8)$ \\
\hline \multicolumn{3}{|c|}{ Presença de morbidade crônica } \\
\hline $\operatorname{Sim}$ & 84 & $78,5(70,6-86,4)$ \\
\hline Não & 23 & $21,5(13,6-29,4)$ \\
\hline \multicolumn{3}{|c|}{ Número de morbidades } \\
\hline Nenhuma & 23 & $21,5(13,6-29,4)$ \\
\hline Até 1 & 51 & $47,7(38,0-57,3)$ \\
\hline 2 ou mais & 33 & $30,8(21,9-39,7)$ \\
\hline \multicolumn{3}{|c|}{ Autoavaliação do estado de saúde } \\
\hline Muito boa & 4 & $3,7(0,1-7,4)$ \\
\hline Boa & 29 & $27,1(18,5-35,7)$ \\
\hline Regular & 56 & $52,3(42,7-62,0)$ \\
\hline Ruim & 17 & $15,9(8,8-22,9)$ \\
\hline Muito ruim & 1 & $0,9(-0,9-2,8)$ \\
\hline Total & 107 & 100,0 \\
\hline
\end{tabular}

IC95\%: intervalo de 95\% de confiança.

* Informação ausente para renda em 6 casos. 
Em relação às variáveis de morbidade, a maioria dos indivíduos entrevistados era portador de diabetes tipo 2 (76,6\%; IC95\%: 68,5-84,8). A maior parte também relatou a presença de outras doenças crônicas além de diabetes (78,5\%; IC95\%: 70,6-86,4), tais como: hipertensão arterial, hipotireoidismo, insuficiência renal, cardiopatia, dentre outras. A maioria relatou possuir um estado de saúde regular, ruim ou muito ruim (69,2\%; IC95\%: 60,3-78,1) (Tabela 1).

A confiabilidade do questionário HLS-EU-BR foi avaliada por meio do coeficiente alfa de Cronbach e obteve os seguintes resultados: 0,72 para a dimensão de promoção de saúde (health promotion), 0,73 para a dimensão de prevenção de doenças (disease prevention) e 0,77 para a dimensão de cuidado ou assistência à saúde (healthcare). Para o questionário como um todo (47 itens), a consistência interna calculada foi de 0,88 . Portanto, o questionário foi considerado confiável em cada uma de suas dimensões e apresentou boa confiabilidade para o conjunto das 47 perguntas.

Foi calculado o índice de LS, obtido a partir das respostas ao questionário aplicado HLS-EU-BR. Observou-se que mais da metade dos 107 pacientes entrevistados tiveram níveis de literacia considerados problemáticos (53,1\%; IC95\%: 43,0-63,3), seguidos por 40 indivíduos que tiveram níveis ainda mais baixos de LS, considerados inadequados (42\%; IC95\%: 32,0-52,0). Apenas 5 indivíduos (5,2\%; IC95\%: 0,7-9,7), nessa população, tiveram nível considerado bom para LS (suficiente ou excelente).

No modelo de regressão ordinal simples, observa-se que ser do sexo masculino aumentou em quase 3 vezes a chance de ter um nível de LS excelente $(\mathrm{p}=0,03)$. Por outro lado, ter um nível de escolaridade mais baixo (menos de 8 anos de estudo) reduziu em $70 \%$ a chance de ter um bom nível de LS $(\mathrm{p}=0,005)$. O aumento da idade esteve associado progressivamente a uma menor chance de possuir um bom nível de LS. Indivíduos com idade entre 46 e 64 anos tiveram 72\% menos chance de ter um nível de LS excelente ( $\mathrm{p}=0,036)$, comparativamente aos indivíduos com até 45 anos. Já os indivíduos com 65 anos ou mais tiveram chance 76\% menor de ter um nível de LS excelente ( $\mathrm{p}=0,026$ ), comparativamente aos indivíduos com até 45 anos. As variáveis cor da pele e renda per capita não tiveram efeito significativo sobre os níveis de LS nesta amostra (Tabela 2).

\section{Tabela 2}

Modelo de regressão ordinal simples para as variáveis sociodemográficas (sexo, idade, cor da pele, escolaridade e renda). Rio de Janeiro, Brasil, 2018.

\begin{tabular}{|c|c|c|c|}
\hline Variáveis & OR bruto & IC95\% & Valor de $p$ \\
\hline \multicolumn{4}{|l|}{ Sexo } \\
\hline Feminino & 1,00 & & \\
\hline Masculino & 2,87 & $1,12-7,32$ & 0,028 * \\
\hline \multicolumn{4}{|l|}{ Idade (anos) } \\
\hline Até 45 & 1,00 & & \\
\hline $46-64$ & 0,28 & $0,08-0,92$ & 0,036 * \\
\hline 65 ou mais & 0,24 & $0,07-0,85$ & 0,026 * \\
\hline \multicolumn{4}{|l|}{ Cor da pele } \\
\hline Branca & 1,00 & & \\
\hline Parda & 0,62 & $0,26-1,46$ & 0,270 \\
\hline Preta & 0,99 & $0,29-3,37$ & 0,993 \\
\hline \multicolumn{4}{|c|}{ Escolaridade (anos de estudo) } \\
\hline Menos de 8 & 0,30 & $0,13-0,69$ & 0,005 * \\
\hline 8 ou mais & 1,00 & & \\
\hline \multicolumn{4}{|c|}{ Renda (salários mínimos) } \\
\hline$>2$ & 1,49 & $0,48-4,61$ & 0,485 \\
\hline$>1$ até 2 & 1,17 & $0,43-3,21$ & 0,754 \\
\hline Até 1 & 1,00 & & \\
\hline
\end{tabular}

IC95\%: intervalo de 95\% de confiança; OR: odds ratio.

* Estatisticamente significativo $(p<0,05)$. 
Em relação às variáveis de morbidade (tipo de diabetes, presença de outra morbidade crônica além de diabetes, número de morbidades crônicas e autoavaliação do estado de saúde), verifica-se que os indivíduos portadores de diabetes tipo 2 (não insulinodependentes) apresentaram $65 \%$ menos chance de ter um nível de LS excelente, comparativamente aos indivíduos portadores de diabetes tipo 1 ( $\mathrm{p}=$ 0,04). Também foi observado que os indivíduos que tiveram uma pior avaliação do seu estado de saúde tiveram 70\% menos chance de ter um bom nível de LS, comparativamente àqueles com uma boa percepção do seu estado de saúde (OR = 0,30; IC95\%: 0,11-0,79; p = 0,015). Em relação às demais variáveis de morbidade, não houve efeito estatisticamente significativo sobre o desfecho LS (Tabela 3).

No modelo ajustado, ao serem incluídas simultaneamente as variáveis que apresentaram significância estatística (Tabelas 2 e 3), apenas a escolaridade permaneceu estatisticamente significativa em relação aos seus efeitos sobre os níveis de LS, como pode ser observado na Tabela 4, na qual são comparadas as OR brutos e ajustados para todas as variáveis.

\section{Discussão}

A abordagem conceitual de Nutbeam 12 para a LS possui origem na saúde pública e na promoção da saúde. Pressupõe a compreensão do papel da educação e da comunicação no desenvolvimento de competências que serão necessárias a diferentes tipos de ações de saúde (sejam elas em nível individual, social ou ambiental), capacitando os indivíduos a exercerem maior controle sobre a sua saúde e os seus determinantes. Segundo essa perspectiva, a LS é mais do que apenas ser capaz de ler rótulos, panfletos ou anotações de saúde. É um recurso a ser construído e, ao promover o acesso das pessoas à informação sobre saúde e capacitá-las a utilizar essa informação de forma eficiente, torna-se uma ferramenta de empoderamento ${ }^{14}$. Nesta abordagem, foi utilizado o modelo proposto por Sorensen et al. 13, que deu origem ao questionário HLS-EU-Q, diferentemente dos questionários sobre literacia funcional, que se baseiam na abordagem da LS vista como um fator de risco relacionada a desfechos em saúde.

\section{Tabela 3}

Modelo de regressão ordinal simples para as variáveis de morbidade (tipo do diabetes, presença de outra morbidade crônica além do diabetes, número de morbidades crônicas e autoavaliação do estado de saúde). Rio de Janeiro, Brasil, 2018.

\begin{tabular}{|c|c|c|c|}
\hline Variáveis & OR bruto & $\mathrm{IC95 \%}$ & Valor de $p$ \\
\hline \multicolumn{4}{|c|}{ Tipo de diabetes } \\
\hline Tipo 1 & 1,00 & & \\
\hline Tipo 2 & 0,35 & $0,13-0,95$ & 0,039 * \\
\hline \multicolumn{4}{|c|}{ Presença de morbidade crônica além do diabetes mellitus } \\
\hline Sim & 1,00 & & \\
\hline Não & 2,65 & $0,86-8,18$ & 0,089 \\
\hline \multicolumn{4}{|c|}{ Número de morbidades } \\
\hline Nenhuma & 1,00 & & \\
\hline Até 1 & 0,36 & $0,11-1,17$ & 0,089 \\
\hline 2 ou mais & 0,41 & $0,11-1,44$ & 0,163 \\
\hline \multicolumn{4}{|c|}{ Autoavaliação do estado de saúde } \\
\hline Boa ** & 1,00 & & \\
\hline Ruim *** & 0,30 & $0,11-0,79$ & 0,015 * \\
\hline
\end{tabular}

IC95\%: intervalo de 95\% de confiança; OR: odds ratio.

* Estatisticamente significativo;

** Muito boa e Boa;

*** Regular, Ruim e Muito ruim. 
Tabela 4

Modelo de regressão ordinal múltipla (ajustado) para as variáveis sociodemográficas (sexo, idade, escolaridade) e de morbidade (autoavaliação do estado de saúde e tipo de diabetes). Rio de Janeiro, Brasil, 2018.

\begin{tabular}{|c|c|c|c|c|c|c|}
\hline Variáveis & OR bruto & IC95\% & Valor de p & $\begin{array}{c}\text { OR } \\
\text { ajustado }\end{array}$ & IC95\% & Valor de p \\
\hline \multicolumn{7}{|l|}{ Sexo } \\
\hline Feminino & 1,00 & & & 1,00 & & \\
\hline Masculino & 2,87 & $1,12-7,32$ & 0,028 * & 1,95 & $0,71-5,35$ & 0,192 \\
\hline \multicolumn{7}{|l|}{ Idade (anos) } \\
\hline Até 45 & 1,00 & & & 1,00 & & \\
\hline $46-64$ & 0,28 & $0,08-0,92$ & 0,036 * & 0,37 & $0,10-1,38$ & 0,138 \\
\hline 65 ou mais & 0,24 & $0,07-0,85$ & $0,026 *$ & 0,42 & $0,09-1,87$ & 0,254 \\
\hline \multicolumn{7}{|c|}{ Escolaridade (anos de estudo) } \\
\hline Menos de 8 & 0,30 & $0,13-0,69$ & $0,005 *$ & 0,41 & $0,17-0,98$ & 0,045 * \\
\hline 8 ou mais & 1,00 & & & 1,00 & & \\
\hline \multicolumn{7}{|c|}{ Autoavaliação do estado de saúde } \\
\hline Ruim ** & 0,30 & $0,11-0,79$ & 0,015 * & 0,47 & $0,16-1,34$ & 0,155 \\
\hline Boa *** & 1,00 & & & 1,00 & & \\
\hline \multicolumn{7}{|c|}{ Tipo de diabetes } \\
\hline Tipo 2 & 0,35 & $0,13-0,95$ & 0,039 * & 0,83 & $0,24-2,82$ & 0,763 \\
\hline Tipo 1 & 1,00 & & & 1,00 & & \\
\hline
\end{tabular}

IC95\%: intervalo de 95\% de confiança; OR: odds ratio.

* Estatisticamente significativo;

** Regular, Ruim e Muito ruim;

$\star \star *$ Muito boa e Boa.

O presente estudo considerou o nível de LS, obtido a partir do escore do questionário HLS-EU-BR, como variável dependente e buscou testar a sua associação com características sociodemográficas e de morbidade que vêm demonstrando associação com LS na literatura, com o objetivo de identificar os grupos mais vulneráveis ou de maior risco de ter um baixo nível de LS. Não foram encontrados, na literatura, estudos que tenham utilizado o instrumento HLS-EU-Q em populações de diabéticos. No Brasil e em outros países, os estudos encontrados nesses pacientes utilizaram instrumentos que medem a literacia funcional, como o TOFHLA ou o Short-Assessment of Health Literacy for Speaking Adults (SAHLSA) 4,5,22,25,26,27,28,29. O único estudo encontrado que aplicou o questionário HLS-EU-BR avaliou uma população, no Rio Grande do Sul, composta por indivíduos com altos níveis de escolaridade, entre universitários e professores 30 .

O presente estudo avaliou portadores de diabetes, uma doença crônica de elevada morbimortalidade, cuja incidência vem crescendo, e as suas complicações associadas acarretam elevado impacto nos custos dos sistemas de saúde 5,26. No Brasil, dados obtidos a partir de um inquérito de base populacional, estimam que a sua prevalência seja em torno de 7,5\% 31. Considera-se importante a avaliação do nível de LS nessa população, visto que se trata de uma doença com elevada complexidade em seu tratamento e controle, os quais dependem, em grande parte, da adequada compreensão do paciente acerca dos mecanismos da doença, dos esquemas terapêuticos e dos mecanismos de prevenção das complicações 29 .

Estudos publicados na literatura sobre LS vêm demonstrando a associação desse construto com variáveis sociodemográficas, como escolaridade, renda, etnia e idade 8,17,20,32 e também com o estado de saúde, como por meio do indicador da autoavaliação do estado de saúde 20. Evidências mostram que, mesmo após o ajuste pelas variáveis sociodemográficas, a LS está relacionada a múltiplos aspectos da saúde, como: o conhecimento sobre saúde, o estado de saúde e o uso de serviços 8 . Os grupos mais vulneráveis, ou seja, aqueles com maior probabilidade de apresentarem um baixo nível de LS, segundo 
dados da literatura, são: indivíduos em idades mais avançadas, minorias étnicas ou raciais, indivíduos com baixos níveis de escolaridade ou renda e indivíduos com várias doenças associadas 20,32.

Cerca de 94,8\% dos indivíduos apresentaram baixos níveis de LS, ou seja, apenas 5,2\% da população estudada apresentou um bom nível de LS. A elevada proporção de indivíduos com baixos níveis de LS encontrada provavelmente se deve a diversos fatores. Em primeiro lugar, deve-se ao perfil da população estudada: indivíduos diabéticos, usuários de um serviço público de saúde, com baixos níveis de escolaridade e renda e com idades mais avançadas. Todas essas características estão associadas a piores níveis de LS na literatura 8,17,20,27,32.

Um estudo conduzido na Argentina, que avaliou 156 pacientes diabéticos em relação ao controle de diabetes e ao nível de LS, esse último medido por meio do questionário SAHLSA, encontrou uma elevada prevalência de LS inadequada comparativamente a estudos similares $(60,3 \%)$ e atribuiu essa diferença ao baixo nível de escolaridade da população do estudo ${ }^{27}$. Outro estudo, conduzido em pacientes com diabetes tipo 2 nos Estados Unidos, utilizando o instrumento TOFHLA, analisou uma população com baixo nível de escolaridade e renda e, em sua maioria, sem plano de saúde 22 . Esse estudo encontrou uma prevalência de LS inadequada de 51\%, enquanto outros estudos conduzidos em pacientes diabéticos com esse instrumento encontraram prevalências em torno de $25 \% 26,29$. Um estudo brasileiro que avaliou 82 pacientes diabéticos tipo 2 atendidos em um ambulatório de um hospital público, utilizando o instrumento TOFHLA, encontrou prevalência de LS de 66\% 5 .

Em segundo lugar, a elevada proporção de indivíduos com baixos níveis de LS deve-se às características do questionário utilizado. Diferentemente dos questionários que avaliam apenas uma dimensão da LS, o HLS-EU-BR foi concebido em sua versão original para abranger todas as dimensões da LS, a saber: funcional, interativa e crítica 14 . Observa-se que os estudos em que esse questionário foi aplicado, em geral, apresentaram percentuais maiores de LS ruim ou limitada, comparativamente aos estudos que utilizaram questionários de avaliação da literacia funcional 23,24,25,26,29. Além disso, outra importante diferença entre o questionário HLS-EU-BR e os questionários de avaliação da literacia funcional é que o primeiro é autorreferido, ou seja, as suas respostas dependem da percepção dos indivíduos, diferentemente do segundo tipo, que captam medidas avaliadas pelo observador. Acredita-se que esse componente subjetivo dos questionários autorreferidos também possa contribuir para os maiores percentuais de LS ruim ou limitada, além de trazer uma potencial associação com o indicador da autoavaliação do estado de saúde ${ }^{16}$. Estudos que já aplicaram esse instrumento no Brasil e em Portugal encontraram elevados percentuais de LS ruim ou limitada (inadequada e problemática), variando de $54,7 \%$ a $62 \%$ 23,24,30, mesmo em populações sem morbidade crônica. Em estudo realizado no Rio Grande do Sul, o questionário HLS-EU-BR foi aplicado em uma população de 179 indivíduos saudáveis com elevados níveis de escolaridade, entre professores universitários, graduandos e estudantes de pós-graduação. Mesmo nessa população distinta, foi encontrada uma prevalência de LS ruim ou limitada de 54,7\%, ou seja, mais da metade da população do estudo apresentou níveis baixos de LS 30 .

As variáveis sociodemográficas que apresentaram associação significativa com o nível de LS foram: sexo, idade e escolaridade. Indivíduos do sexo masculino, com idades mais jovens e com maior escolaridade tiveram mais chances de terem um nível de LS excelente. Por outro lado, ser do sexo feminino, ter idades mais avançadas e menor escolaridade estiveram associados a piores níveis de LS. Um estudo norte-americano conduzido em 408 pacientes com diabetes tipo 2, que avaliou a LS por meio do instrumento TOFHLA, também demonstrou que indivíduos com níveis inadequados de LS tinham maior probabilidade de serem mais idosos, do sexo feminino e possuírem baixa escolaridade 22. Em dois estudos conduzidos no Brasil em pacientes diabéticos, utilizando o instrumento TOFHLA, idade mais avançada e o baixo nível de escolaridade também estiveram associados a piores resultados para a LS 5,26. Em estudo conduzido na Turquia, a escolaridade esteve associada positivamente à LS, independentemente do instrumento de aferição ${ }^{28}$. Em estudo conduzido na Argentina, a baixa escolaridade também esteve associada a um nível de LS inadequado 27.

No presente estudo, mesmo após o ajuste pelas variáveis sociodemográficas e de morbidade, a variável escolaridade permaneceu estatisticamente significativa em relação ao seu efeito sobre a LS. Ou seja, nessa população, essa foi a característica que esteve mais fortemente relacionada ao nível de LS, com os indivíduos com menor escolaridade apresentando chance $60 \%$ menor de ter um nível de LS considerado excelente, comparativamente aos indivíduos com maior escolaridade (OR ajustado $=0,41$; 
IC95\%: 0,17-0,98; $\mathrm{p}<0,05)$. Nos dois estudos brasileiros conduzidos em pacientes diabéticos que utilizaram o instrumento TOFHLA, também foi encontrada associação estatística entre LS e as variáveis idade e escolaridade, mesmo nos modelos ajustados 5,26. Em estudo realizado na Turquia, nos modelos ajustados, as variáveis renda e escolaridade permaneceram significativas na sua associação com LS para o instrumento REALM, e apenas a escolaridade permaneceu significativa para o instrumento NVS 28.

No único estudo brasileiro que avaliou a LS por meio do questionário HLS-EU-BR, não foram encontradas diferenças nos níveis de LS de acordo com a idade e, em relação ao sexo, diferentemente do presente estudo, as mulheres apresentaram melhores níveis de LS 30. Neste estudo, não foram construídos modelos de regressão, e a escolaridade não foi avaliada, provavelmente por já se tratar de uma população com elevados níveis de escolaridade.

Em relação às variáveis de morbidade, a autoavaliação do estado de saúde e o tipo de diabetes estiveram associados significativamente ao nível de LS. Indivíduos com autoavaliação do estado de saúde ruim tiveram $70 \%$ menos chance de terem um nível de LS considerado excelente. Da mesma forma, os pacientes portadores de diabetes tipo 2 possuíram 65\% menos chance de ter um nível de LS excelente. A associação entre autoavaliação do estado de saúde e o nível de LS vem sendo descrita na literatura, com baixos níveis de LS, sendo associados a uma pior percepção do estado de saúde 20, mas nenhum estudo foi encontrado que tenha testado diretamente essa associação em pacientes portadores de morbidade crônica, especialmente diabetes. No modelo ajustado, para a população do presente estudo, a autoavaliação do estado de saúde deixou de ter um efeito estatisticamente significativo sobre a LS.

A associação entre o diagnóstico autorreferido de diabetes tipo 2 e um pior nível de LS provavelmente se deveu aos piores níveis de escolaridade desses pacientes, comparativamente aos pacientes com diabetes tipo 1. Entre os 25 indivíduos portadores de diabetes tipo 1, 32\% apresentavam menos de 8 anos de estudo, ao passo que, entre os 82 portadores de diabetes tipo 2, 57,3\% apresentavam menos de 8 anos de estudo. No modelo final ajustado, o tipo de diabetes deixou de exercer um efeito estatisticamente significativo sobre a LS, permanecendo significativo apenas o efeito da escolaridade.

O presente estudo verificou um elevado percentual de indivíduos com LS ruim ou limitada (94,8\%). Isso pode ser explicado, a princípio, por duas razões: pelas características específicas da população estudada: indivíduos portadores de uma doença crônica de elevada morbimortalidade, com baixos níveis de escolaridade e renda e com idades mais avançadas (a mediana de idade dessa população foi de 61 anos; IQR: 52-68); e pelo tipo de questionário utilizado para medir LS, um questionário autorreferido, multidimensional, que vem apresentando maiores percentuais de LS ruim ou limitada, comparativamente aos estudos que utilizaram questionários de avaliação da literacia funcional.

De toda a forma, o elevado percentual de indivíduos com LS ruim ou limitada chama a atenção, principalmente por se tratar de indivíduos portadores de uma doença crônica de elevada complexidade, como diabetes. O principal fator que parece estar relacionado com o nível de LS foi o nível de escolaridade. Mesmo após o ajuste, o nível de escolaridade permaneceu significativo em relação ao seu efeito sobre a LS. Indivíduos com menores níveis de escolaridade possuíram significativamente menor chance de terem um nível de LS considerado excelente. Indivíduos portadores de diabetes tipo 2, o tipo mais prevalente na população, apresentaram os piores níveis de escolaridade.

A LS está intimamente ligada à literacia e pressupõe o conhecimento, a motivação e as competências dos indivíduos para acessarem, compreenderem, avaliarem e aplicarem as informações sobre saúde, a fim de fazer julgamentos e tomar decisões na vida diária, relacionadas aos cuidados de saúde, à prevenção de doenças e à promoção de saúde, para manter ou melhorar a sua qualidade de vida durante o curso da vida 13. Portanto, é razoável esperar que o nível de LS deva possuir relação com o nível de escolaridade, apesar de que isso pode não ser a regra, visto que LS e grau de instrução representam conceitos distintos. Na literatura, outros aspectos também vêm se mostrando relacionados com o nível de LS, como idade, cor de pele, renda e autoavaliação do estado de saúde, que também foram avaliados no presente estudo ${ }^{20}$. Nas análises univariadas, foram verificadas associações com o nível de LS para sexo, idade e autoavaliação do estado de saúde. Um estudo norte-americano conduzido em 408 pacientes com diabetes tipo 2 também demonstrou que indivíduos com níveis inadequados de LS tinham maior probabilidade de terem idade mais avançada, de serem do sexo feminino e de possuírem baixa escolaridade 22 .

As limitações deste estudo estão relacionadas ao seu desenho transversal, que impede o estabelecimento de relações de causa-efeito. Além disso, outra limitação importante diz respeito à dificuldade 
de compreensão dos participantes a determinadas perguntas do questionário, identificada durante o trabalho de campo. Este fato sinalizou a necessidade ainda existente, e que pode ser contemplada em estudos futuros, de uma revisão na linguagem do instrumento a fim de facilitar a compreensão dos participantes (como a redução no tamanho das perguntas e maior objetividade e clareza na linguagem), até por se tratar de um instrumento multidimensional e de maior complexidade.

Portanto, pode-se concluir que, nessa população avaliada, a prevalência de LS ruim ou limitada foi bastante elevada. Idades mais avançadas, diabetes tipo 2 e autoavaliação do estado de saúde ruim estiveram associados a níveis mais baixos de LS, mas o principal fator que parece estar relacionado com o nível de LS foi a escolaridade.

\section{Colaboradores}

A. L. B. Pavão contribuiu com a concepção e delineamento do estudo, análises e redação do manuscrito. G. L. Werneck contribuiu com o delineamento do estudo, análises e revisão do manuscrito. L. Saboga-Nunes contribuiu no delineamento do estudo e análise dos dados. R. A. Sousa contribuiu na análise dos dados. Todos os autores aprovaram a versão final.

\section{Informações adicionais}

ORCID: Ana Luiza Braz Pavão (0000-0002-41221796); Guilherme Loureiro Werneck (0000-00031169-1436); Luis Saboga-Nunes (0000-0002-73784438); Rosane Aparecida de Sousa (0000-00025943-4175).

\section{Agradecimentos}

À Coordenação de Aperfeiçoamento de Pessoal de Nível Superior (CAPES) pela bolsa de pós-doutorado.

\section{Referências}

1. Saboga-Nunes L. Literacia para a saúde e a conscientização da cidadania positiva. Referência 2014; III Suppl 11:94-9.

2. Saboga-Nunes L, Freitas O, Cunha M. Renasceres: um modelo para a construção da cidadania em saúde através da literacia para a saúde. Revista Servir 2016; 59:7-16.

3. Saboga-Nunes L. Hermenêutica da literacia em saúde e sua avaliação em Portugal (HLS-EU-PT). In: VIII Congresso Português de Sociologia. 40 anos de democracia(s): progressos, contradições e prospetivas. Lisboa: Associação Portuguesa de Sociologia; 2014. p. 1-15.

4. Apolinario D, Braga RCOP, Magaldi RM, Busse AL, Campora F, Brucki S, et al. Short assessment of health literacy for portuguesespeaking adults. Rev Saúde Pública 2012; 46:702-11.

5. Sampaio HAC, Carioca AAF, Sabry MOD, Santos PM, Coelho MAM, Passamai MPB. Letramento em saúde de diabéticos tipo 2: fatores associados e controle glicêmico. Ciênc Saúde Colet 2015; 20:865-74.

6. Martins NFF, Abreu DPG, Silva BT, Semedo DSRC, Pelzer MT, Ienczak FS. Letramento funcional em saúde e adesão à medicação em idosos: revisão integrativa. Rev Bras Enferm 2017; 70:868-74.

7. Martins AMEB, Almeida ER, Oliveira CC, Pelino JEP, Santos ASF, Costa AS, et al. Alfabetização em saúde bucal: uma revisão da literature. Rev Assoc Paul Cir Dent 2015; 69:328-34.

8. Ad Hoc Committee on Health Literacy for the Council on Scientific Affairs, American Medical Association. Health literacy: report of the council on scientific affairs. JAMA 1999; 281:552-7. 
9. Institute of Medicine. Health literacy: a prescription to end confusion. Washington DC: The National Academies; 2004.

10. Nutbeam D. Health promotion glossary. Health Promot Int 1998; 13:349-64.

11. Pleasant A, Kuruvilla SS. A tale of two health literacies: public health and clinical approaches to health literacy. Health Promot Int 2008; 23:152-9.

12. Nutbeam D. The evolving concept of health literacy. Soc Sci Med 2008; 67:2072-8.

13. Sorensen K, Van den Broucke S, Fullam J, Doyle G, Pelikan J, Slonska Z, et al. Health literacy and public health: a systematic review and integration of definitions and models. BMC Public Health 2012; 12:80.

14. Nutbeam D. Health literacy as a public goal: a challenge for contemporary health education and communication strategies into the $21 \mathrm{st}$ century. Health Promot Int 2000; 15:259-67.

15. Zarcadoolas C, Pleasant A, Greer DS. Understanding health literacy: an expanded model. Health Promot Int 2005; 20:195-203.

16. Pleasant A. Advancing health literacy measurement: a pathway to better health and health system performance. J Health Commun 2014; 19:1481-96.

17. Paasche-Orlow MK, Parker RM, Gazmararian JA, Nielsen-Bohlman LT, Rudd RR. The prevalence of limited health literacy. J Gen Intern Med 2005; 20:175-84.

18. Berkman ND, Sheridan SL, Donahue KE, Halpern DJ, Crotty K. Low health literacy and health outcomes: an updated systematic review. Ann Intern Med 2011; 155:97-107.

19. Baker DW, Parker RM, Williams MV, Clark WS, Nurss J. The relationship of patient reading ability to self-reported health and use of health services. Am J Public Health 1997; 87:1027-30

20. World Health Organization. Health literacy: the solid facts. http://www.euro.who.int/_data/assets/pdf_file/0008/190655/e96854.pdf (acessado em 30/Abr/2019).

21. Brown H, Prisuta R, Jacobs B, Campbell A. Literacy of older adults in America: results from the National Adult Literacy Survey. Washington DC: National Center for Education Statistics, U.S. Department of Education; 1996.

22. Schillinger D, Grumbach K, Piette J, Wang F, Osmond D, Daher C, et al. Association of health literacy with diabetes outcomes. JAMA 2002; 288:475-82.

23. Martins R, Saboga-Nunes L. The challenges of epistemological validation to Brazil of the European Health Literacy Survey (HLS-EU-BR). Aten Primaria 2014; 46(Espec Cong 1):12.
24. Saboga-Nunes L, Sørensen K, Pelikan J, Cunha M, Rodrigues E, Paixão E. Cross-cultural adaptation and validation to Portuguese of the European Health Literacy Survey (HLS-EU-PT). Aten Primaria 2014; 46(Espec Cong 1):13.

25. Apolinario D, Mansur LL, Carthery-Goulart MT, Brucki SMD, Nitrini R. Detecting limited health literacy in Brazil: development of a multidimensional screening tool. Health Promot Int 2013; 29:5-14.

26. Castro SH, Brito GNO, Gomes MB. Health literacy skills in type 2 diabetes mellitus outpatients from an university-affiliated hospital in Rio de Janeiro, Brazil. Diabetol Metab Syndr 2014; 6:126.

27. Bolivar AA, Lanteri ME, Cícero CY, Pérez A, Puchulu FM, Mejía R. Alfabetización en salud y control de la diabetes en pacientes de un hospital universitario de Argentina. Medicina (B. Aires) 2017; 77:167-72.

28. Keles OR, Ilicali NI, Ozcan MC, Topsever P. Health literacy in patients with type 2 diabetes and its relationship with glycemic control and sociodemographic factors: preliminary results of a descriptive study. Prim Care Diabetes 2013; 7:81-2.

29. Bohanny W, Wu SFV, Liu CY, Yeh SH, Tsay SL, Wang TJ. Health literacy, self-efficacy, and self-care behaviors in patients with type 2 diabetes mellitus. J Am Assoc Nurse Pract 2013; 25:495-502.

30. Carvalho GS, Araújo MCP, Boff ETO, Tracana RB, Saboga-Nunes L. European health literacy scale (HLS-EU-BR) applied in a Brazilian higher education population of Rio Grande do Sul (RS). In: Lavanen J, Juuti K, Lampiselka J, Uitto A, Hahl K, editores. Electronic proceedings of the ESERA 2015 Conference. Helsinki: University of Helsinki; 2016. p. 1289-95.

31. Flor LS, Campos MR. Prevalência de diabetes mellitus e fatores associados na população adulta brasileira: evidências de um inquérito de base populacional. Rev Bras Epidemiol 2017; 20:16-29.

32. U.S. Department of Health and Human Services. Quick guide to health literacy. https:// health.gov/communication/literacy/quickgui de/ (acessado em 30/Abr/2019). 


\section{Abstract}

Health literacy (HL) assumes individuals' knowledge, motivation, and competencies to access, understand, evaluate, and apply health information to make judgments and decisions in daily life, related to healthcare, prevention of diseases, and health promotion to maintain or improve quality of life. The study aimed to measure the level of $H L$ and associated factors: sex, age, schooling, income, skin color, self-rated health status, type of diabetes, and presence of comorbidities. The authors assessed 107 adults with diabetes followed at a public outpatient clinic in the city of Rio de Janeiro, Brazil. The sample did not include first-time patients, patients without a diagnosis of diabetes, or with visual or hearing impairment. HL was assessed with the Brazilian version of the European Health Literacy Survey (HLS-EU-BR). Simple and multiple ordinal logistic regression models were constructed, considering four levels of $H L$ as the dependent variables. The associations were expressed as odds ratios (OR). Approximately 95\% of the sample showed poor or limited HL (94.8\%; 95\%CI: 90.3-99.3). Female gender, older age, and lower schooling were associated with lower odds of excellent HL. In the adjusted model, only schooling remained statistically significant in its effect on HL (adjusted OR =0.41; 95\%CI: 0.17-0.98; $p<0.05)$. Schooling was the characteristic most strongly related to level of $H L$.

Surveys and Questionnaires; Diabetes Mellitus; Health Literacy

\section{Resumen}

La alfabetización en salud (AS), o cultura en salud, presupone el conocimiento, la motivación y las competencias de los individuos para que accedan, comprendan, evalúen y apliquen información sobre salud, con el fin de realizar juicios y tomar decisiones en la vida diaria, relacionadas con los cuidados de salud, prevención de enfermedades, $y$ con la promoción de salud, para mantener o mejorar su calidad de vida. El objetivo fue medir el nivel de AS y sus factores asociados: sexo, edad, escolaridad, renta, color de piel, autoevaluación del estado de salud, tipo de diabetes y presencia de comorbilidades. Se evaluaron a 107 adultos pacientes diabéticos, a quienes se les realizó un seguimiento en un ambulatorio público, en la ciudad de Río de Janeiro, Brasil. No se incluyeron pacientes que fueron la primera vez, pacientes sin diagnóstico de diabetes o con limitaciones visuales o auditivas. La AS se evaluó mediante la versión brasileña del cuestionario European Health Literacy Survey (HLS-EU-BR). Se construyeron modelos de regresión logística ordinal simple y múltiple, considerándose como variable dependiente los cuatro niveles de AS. Las asociaciones se expresaron en forma de odds ratio (OR). Cerca de un 95\% de la muestra presentó un nivel de AS malo o limitado (94,8\%; IC95\%: 90,3-99,3). Sexo femenino, edades más avanzadas, y menor escolaridad estuvieron asociados con una menor oportunidad de tener un nivel de AS excelente. En el modelo ajustado, solamente la variable escolaridad fue estadisticamente significativa, en relación con su efecto sobre la AS (OR ajustado = 0,41; IC95\%: 0,17-0,98; $p<0,05)$. La escolaridad fue la característica que estuvo más fuertemente relacionada con el nivel de AS.

Encuestas y Cuestionarios; Diabetes Mellitus; Alfabetización en Salud
Recebido em 05/Ago/2020

Versão final reapresentada em 27/Nov/2020

Aprovado em 26/Jan/2021 